\title{
Evaluating Cost and Performance for Improved Meningitis Disease Surveillance in Chad
}

\author{
Ngozi Erondu* and Ulla K. Griffiths \\ London School of Hygiene and Tropical Medicine, London, United Kingdom
}

\section{Objective}

This presentation shares findings of a cost and performance evaluation of the meningitis surveillance system in Chad. We will also present methods used to design an operational standard for meningitis surveillance in Chad and a cost extrapolation model for other meningitis affected countries in sub-Saharan Africa.

\section{Introduction}

The costs of addressing specific needs to improve surveillance systems in sub-Saharan Africa are often unknown. For centuries meningococcal meningitis epidemics have occurred every few years in the Sahelian and sub-sahel regions of Africa also referred to as the "African meningitis belt". A serogroup A meningococcal conjugate vaccine, MenAfriVac ${ }^{\circledR}$, was licensed in 2009 and introduced in phases through mass immunization campaigns for all 1-29 year olds. The long term health impact of MenAfriVac ${ }^{\circledR}$ can only be determined if strong disease surveillance is in place. The objective was to estimate the costs and assess the performance of meningitis surveillance in Chad to determine resources needed for implementing a district casebased surveillance strategy.

\section{Methods}

Performance and cost data were obtained through structured interviews with laboratory and clinical staff in seven districts and at the national level. In each district, three primary care facilities and one district laboratory was included. Surveillance officials at intermediate and national levels were also interviewed. Resource quantities and respective unit costs were collected and categorized according to core surveillance functions (detect, report, analysis, feedback, investigation, and response), and support activities (training, supervision, communication, and co-ordination). Unit costs were collected from numerous sources, including financial records of the Ministry of Health and international partners. Based on the data, an upgraded surveillance standard was developed by 1) defining the gaps in the meningitis surveillance system, 2) identifying the needs at each jurisdictional level, 3) constructing a feasible operational standard and 4) estimating the cost of implementing the standard.

\section{Results}

Optimal surveillance was severely hampered by limited resources, including missing laboratory materials and inadequate staff. In 14 of the facilities, confusion and misinterpretation of a national policy translated into missed opportunities for staff to perform lumbar punctures on patients presenting with suspected meningitis; patients were referred to district hospitals in the remaining seven.

Missing and unreliable data affected case detection and reporting; in three of the districts, no meningitis cases were reported during 2012. In the other four districts, reported cases varied between 43 and 232, equivalent to between 11 and 89 per 100,000 populations. 9\% of specimens were sent to the national laboratory for confirmation and $4 \%$ of probable meningitis cases had a known outcome reported. In facilities with no detected cases, resources spent on surveillance were marginal. Costs per detected cases amounted to US\$ 49. Costs of lumbar puncture comprised $43 \%$ and laboratory analysis $41 \%$ of total costs.

Several features were included in the upgraded surveillance model such as, periodic surveillance training for relevant staff, motorbikes for district surveillance officers, resources for patient transfer, and a courier system for specimen transport from district to national laboratories.

\section{Conclusions}

The study findings provide cost estimates that were used to recommend a feasible and effective meningitis surveillance strategy for Chad. While investments are needed across the system in many African meningitis belt countries, a systematic approach that assesses performance gaps and highlights areas of optimization can provide a more sustainable solution for integrated disease surveillance.

\section{Keywords}

Costing; Monitoring and Evaluation; Meningitis; MenAfriVac; Chad

\section{Acknowledgments}

The authors would like to acknowledge and thank our Chadian collaborator, Centre de Support en Santé Internationale. We also are grateful for the contributions by the World Health Organization, Agence de Médecine Préventive, and our funder, the Bill and Melinda Gates Foundation.

\section{References}

Daugla DM, Gami JP, Gamougam K, Naibei N, Mbainadji L, Narbe $\mathrm{M}$, et al. Effect of a serogroup A meningococcal conjugate vaccine (PsA-TT) on serogroup A meningococcal meningitis and carriage in Chad: a community study [corrected]. Lancet. 2014;383(9911):40-7.

WHO. Epidemic Meningitis Surveillance in the African Meningitis Belt: Deciding on the most appropriate approach. Geneva: 2014.

Kasolo F, Roungou J.B., Perry, H. Technical guidelines for integrated disease surveillance and response in the African region. WHO and CDC, October 2010.

\author{
*Ngozi Erondu \\ E-mail: ngozierondu@gmail.com
}

